\title{
Chromosome pairing and chiasma formation in spermatocytes and oocytes of Dendrocoelum lacteum (Turbellaria, Tricladida); a cytogenetical and ultrastructural study
}

\author{
G. H. Jones and
}

J. A. Croft
School of Biological Sciences, University of Birmingham, Birmingham B15 27T, U.K.

The hermaphroditic flatworm Dendrocoelum lacteum shows a very pronounced sex difference in chiasma frequency; oocytes have 66 per cent more chiasmata than spermatocytes. The ultrastructural basis of this difference was investigated by three-dimensional reconstruction of synaptonemal complexes from ten oocytes and ten spermatocytes at pachytene and smaller numbers of zygotene nuclei. Oocyte nuclei at pachytene are larger and have longer synaptonemal complexes than the equivalent spermatocyte stage. The difference in synaptonemal complex length (60 per cent) corresponds very closely to the difference in chiasma frequency shown by oocytes and spermatocytes and suggests that synaptonemal complex (SC) length may contribute to the regulation of chiasma frequency.

Recombination nodules were observed in both spermatocytes and oocytes but their quantitation was unreliable because they are small and differentiation from the unusually dense and prominent central region is difficult. Analysis of the few zygotene nuclei available shows that pairing is predominantly proterminal in both spermatocytes and oocytes, but oocytes may have several additional interstitial initiations. These extra initiations are likely to be necessary for efficient pairing of the longer oocyte chromosomes.

\section{INTRODUCTION}

Dendrocoelum lacteum is a common freshwater planarian which is very widely distributed throughout Europe in all types of water bodies (Ball and Reynoldson, 1981). It has a relatively long but sporadic history of cytogenetical investigation (Matthieson, 1904; Arnold, 1909; Gelei, 1913, 1921, 1922; Aeppli, 1951; Pastor and Callan, 1952; Benazzi and Pochini, 1959). Its somatic chromosome number of 14 was first accurately determined by Gelei (1913), but there has not always been agreement on this point (see Benazzi and Benazzi-Lentati, 1976 for details). Gelei (1913, 1921, 1922) also recognised the value of this species for the cytogenetical investigation of meiosis and conducted a detailed analysis of the course of chromosome pairing in oocytes which became a classical study in this field. Gelei's studies not only vindicated the parasynapsis (side-by-side) hypothesis of chromosome pairing but also confirmed the homologous nature of this pairing. $\mathrm{He}$ also recognised the phenomenon of chromosome interlocking during zygotene, an aspect of chromosome behaviour whose significance has only recently been reappreciated.

The hermaphroditic nature of $D$. lacteum was exploited by Pastor and Callan (1952) in a comparative study of chiasmata in later stages of spermatogenesis and oogenesis. They found that chiasma frequency in oocytes was much higher than in spermatocytes, thus providing one of the earliest as well as most dramatic examples of sex differences in chiasma frequency. The aim of the present study was to investigate the ultrastructural basis of this marked meiotic sexual dimorphism by extending the analysis of meiotic pairing in this species to the ultrastructural investigation of synaptonemal complexes (SCs) in oocytes and spermatocytes.

\section{MATERIALS AND METHODS}

Adult sexually mature specimens of $D$. lacteum were collected from Winterbourne Lake, Edgbaston, Birmingham between November and April, 
when oogenesis and spermatogenesis were most prevalent (Pastor and Callan, 1952).

\section{Electron microscopy}

Entire animals (1-2 cm long) were fixed in 4 per cent glutaraldehyde in phosphate buffer at $p \mathrm{H} \mathrm{7 \cdot 2}$ for 30 minutes at room temperature. The animals were then cut into small pieces $(2-3 \mathrm{~mm})$ and then returned to the fixative for a further $3 \frac{1}{2}$ hours. Some specimens were treated with 2 per cent osmium tetroxide in phosphate buffer at $p \mathrm{H} 7 \cdot 2$ for 2 hours at room temperature and dehydrated through an alcohol series. For other specimens the osmium tetroxide treatment was omitted; instead they were passed directly through an alcohol series and then treated with 1 per cent alcoholic phosphotungstic acid (PTA) for 16 hours at room temperature. Following these treatments, all specimens were embedded in Epon 812 and polymerised at $60^{\circ} \mathrm{C}$ for 20 hours. Serial thin sections $(100 \mathrm{~nm})$ were cut on a Reichert-Jung ultramicrotome equipped with a diamond knife. Serial ribbons of sections were transferred to Formvar coated single slot grids using the indirect method of Wells (1974). Sections that had been previously treated with osmium tetroxide were further stained with 1 per cent aqueous uranyl acetate at $40^{\circ} \mathrm{C}$ for 90 minutes and post stained with lead citrate for 2 minutes at $20^{\circ} \mathrm{C}$ in an LKB 2168 Ultrostainer. Sections of PTA treated specimens required no further staining. Serial sections were examined and photographed using a JEOL 1200EX electron microscope. Individual SCs were traced from the series onto transparent acetate overlays and SC lengths were measured using a Summagraphics digitising tablet linked to a BBC microcomputer which was programmed to include section thickness during the reconstruction procedure.

\section{Light microscopy}

The mitotic karyotype was ascertained from orcein-stained squash preparations of young embryos. Newly laid coccoons, which contain several embryos, were collected and kept in pond water at $15^{\circ} \mathrm{C}$ for $6-8$ days. At this stage the coccoon shells were punctured with a sharp needle and the coccoons were treated with 0.05 per cent colchicine for 3 hours before fixing in $3: 1$ acetic alcohol overnight. Lactopropionic orcein-stained squash preparations were then made using small portions of coccoon contents.

Chiasma analysis was carried out on Feulgen and orcein-stained squash preparations of sper- matocytes and oocytes. Entire animals were fixed in $3: 1$ acetic alcohol and then Feulgen stained to facilitate the location of testis and ovary material. Small portions of the animals containing either testis or ovary material were then squashed in lactopropionic orcein.

\section{RESULTS}

\section{Light Microscopy}

\section{Somatic karyotype}

The diploid somatic karyotype of the D. lacteum population employed in this study consists of 14 chromosomes containing seven pairs which can be identified individually on the basis of overall length combined with arm ratio (see fig. 1). The somatic chromosome number of 14 confirms the majority of previous investigations and does not accord with somatic chromosome counts of 16 reported by some investigators (see table 2(b) of Benazzi and Benazzi-Lentati, 1976). Published details of the somatic karyotype of D. lacteum are scarce (e.g., Dahm, 1961; Melander, 1963) and none of them agree completely with the karyotype observed in the present investigation. Whether this reflects intra-specific chromosome polymorphism remains an open question.

\section{Meiotic chromosomes and chiasmata}

All stages of spermatocyte meiosis are readily observed in Feulgen and orcein-stained squash preparations of testis material. The chiasmata of spermatocytes are most conveniently scored in nuclei at metaphase I (fig. 2(a))). Most bivalents are easily identifiable at this stage, with the exception of chromosomes 5 and 6 which are sometimes indistinguishable. Ovarian squash preparations yield numerous prophase I oocyte nuclei including late stages (diplotene to diakinesis) in which seven relatively uncondensed bivalents are visible (fig. 2(b)). Because centromeres are not generally discernible at these stages, bivalent identification is not possible in oocytes.

As part of this study, an extensive survey of chiasma variation in the Winterbourne Lake population was undertaken. Several animals were sampled on each of three different collection dates (December, January, February) in order to determine whether any seasonal variation in chiasma frequency occurs and also to ascertain the extent of inter-individual chiasma variation (table 1). The chiasma frequencies of both spermatocytes and 


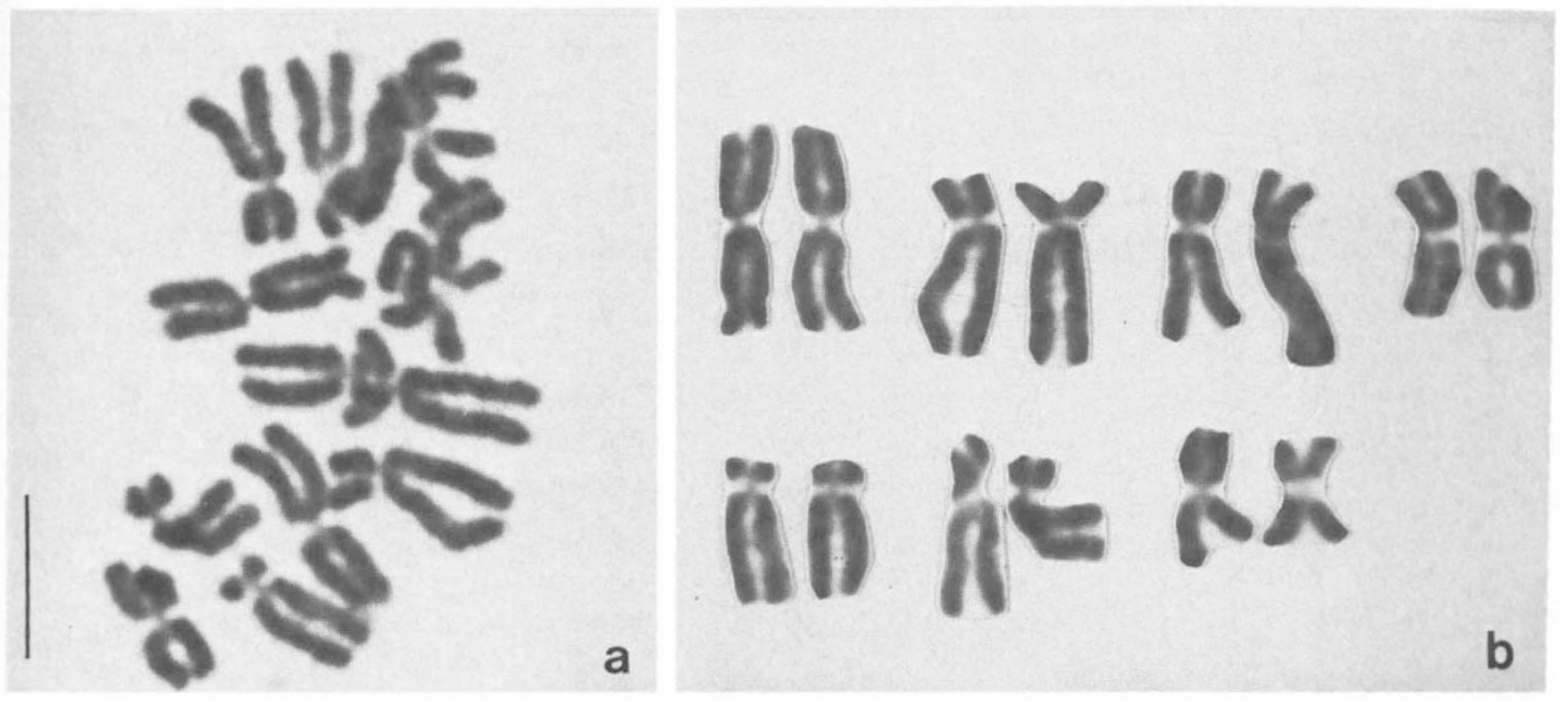

Figure 1 Colchicine treated embryonic mitotic metaphase of Dendrocoelum lacteum (a) and the mitotic karyotype derived from the same cell (b). (Bar $=5 \mu \mathrm{m})$.

oocytes are slightly higher in collection $\mathrm{C}$ (February) as compared to the other two collections, but this difference is only significant for oocytes and then only marginally so $(P<0.05)$. Significant inter-individual variation occurs for both oocytes and spermatocytes. In agreement with the earlier finding by Pastor and Callan (1952), the chiasma frequencies of oocytes and sper- matocytes in the same individuals are positively correlated (fig. 3); the regression of oocyte chiasma frequency on spermatocyte chiasma frequency is significant $\left(F_{(1,15)}=7 \cdot 402 ; P<0.05\right)$. However, by far the most striking source of chiasma variation in this study is the difference between oocytes and spermatocytes. The mean chiasma frequency of oocytes in this population is $19 \cdot 31$ (range $17 \cdot 25$ to

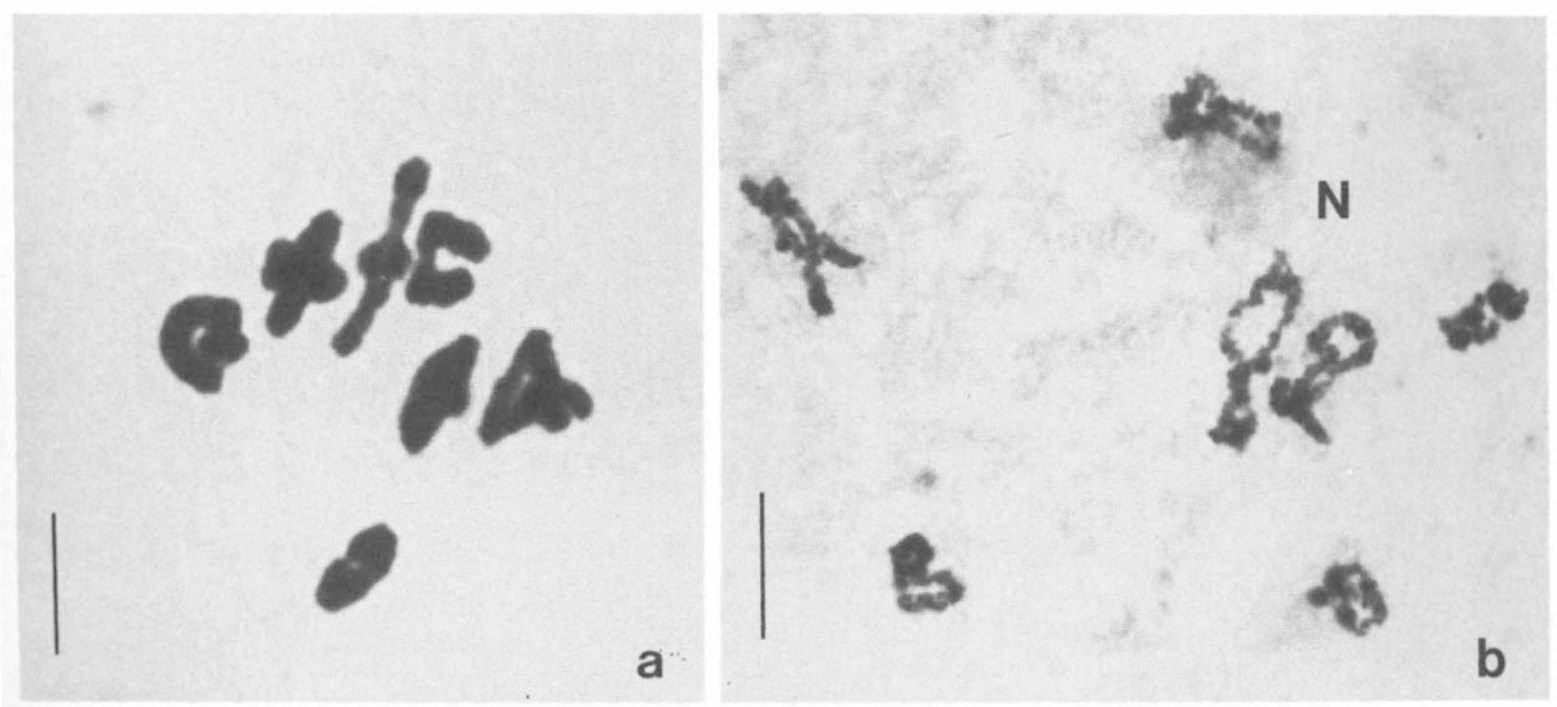

Figure 2 Meiotic chromosomes of Dendrocoelum lacteum. (a) Spermatocyte first metaphase with seven condensed bivalents containing 12 chiasmata. $(B a r=5 \mu \mathrm{m})$. (b) Oocyte diakinesis with seven relatively uncondensed bivalents containing 18 chiasmata. The nucleolus $(N)$ is associated with two of the oocyte bivalents. (Bar $=10 \mu \mathrm{m}$ ). 
Table 1 A summary of mean chiasma frequencies $(\bar{x})$ and their standard deviations (s.d.) of spermatocytes and oocytes from Dendrocoelum lacteum individuals collected on three different occasions from Winterbourne Pool, Edgbaston, Birmingham. $\mathrm{A}=$ December, $\mathrm{B}=$ January, $\mathrm{C}=$ February. $n=$ number of cells examined

\begin{tabular}{|c|c|c|c|c|c|c|c|}
\hline \multirow[b]{2}{*}{ Collections } & \multirow[b]{2}{*}{ Individuals } & \multicolumn{3}{|l|}{$q$} & \multicolumn{3}{|l|}{$\delta$} \\
\hline & & $\bar{x}$ & $n$ & s.d. & $\bar{x}$ & $n$ & s.d. \\
\hline \multirow[t]{6}{*}{ A } & 1 & $18 \cdot 50$ & 2 & $0 \cdot 71$ & $11 \cdot 33$ & 15 & 1.05 \\
\hline & 2 & $18 \cdot 80$ & 10 & $1 \cdot 32$ & $10 \cdot 87$ & 15 & 1.06 \\
\hline & 3 & $17 \cdot 25$ & 4 & $1 \cdot 50$ & $10 \cdot 80$ & 15 & $1 \cdot 15$ \\
\hline & 4 & $18 \cdot 71$ & 7 & 1.60 & $12 \cdot 27$ & 15 & 0.80 \\
\hline & 5 & $20 \cdot 00$ & 8 & 1.77 & $11 \cdot 13$ & 15 & 0.92 \\
\hline & 6 & $18 \cdot 80$ & 10 & $2 \cdot 15$ & $12 \cdot 40$ & 15 & $1 \cdot 35$ \\
\hline \multirow[t]{6}{*}{ B } & 1 & $21 \cdot 00$ & 3 & $2 \cdot 65$ & $12 \cdot 53$ & 15 & $1 \cdot 19$ \\
\hline & 2 & $19 \cdot 00$ & 5 & 1.41 & $11 \cdot 14$ & 7 & 1.07 \\
\hline & 3 & $20 \cdot 13$ & 8 & $1 \cdot 36$ & $12 \cdot 20$ & 15 & $1 \cdot 21$ \\
\hline & 4 & $18 \cdot 60$ & 10 & $1 \cdot 71$ & $10 \cdot 87$ & 15 & $1 \cdot 25$ \\
\hline & 5 & $16 \cdot 90$ & 10 & $1 \cdot 37$ & $10 \cdot 33$ & 15 & $1 \cdot 11$ \\
\hline & 6 & $17 \cdot 10$ & 10 & $1 \cdot 60$ & $11 \cdot 47$ & 15 & $0 \cdot 74$ \\
\hline \multirow[t]{5}{*}{$\mathrm{C}$} & 1 & $20 \cdot 80$ & 10 & $2 \cdot 49$ & $12 \cdot 00$ & 15 & 1.51 \\
\hline & 2 & $22 \cdot 75$ & 8 & 1.98 & $11 \cdot 73$ & 15 & 0.88 \\
\hline & 3 & $18 \cdot 80$ & 5 & $2 \cdot 28$ & $12 \cdot 13$ & 15 & $1 \cdot 55$ \\
\hline & 4 & $21 \cdot 75$ & 8 & $1 \cdot 28$ & $12 \cdot 47$ & 15 & 1.64 \\
\hline & 5 & $19 \cdot 40$ & 5 & $2 \cdot 07$ & 11.93 & 15 & 1.03 \\
\hline
\end{tabular}

$22.75)$ while that of spermatocytes is much less at 11.62 (range 10.33 to 12.53 ), fully confirming the observations of Pastor and Callan (1952).

Chiasma distribution is difficult to quantify accurately in this species because of the small chromosomes and the lack of centromere visibility in late prophase I oocytes. Nevertheless a general description of chiasma distribution is possible based on visual assignment of chiasmata to arbitrary sub-divisions of bivalents. In both spermatocytes and oocytes chiasmata are widely distributed along bivalents, but with a tendency to pro-terminal localisation. Pro-terminal segments of bivalents, occupying 40 per cent of total chromosome length ( 20 per cent at each end) contain 70 and 65 per cent, respectively, of all chiasmata in spermatocytes and oocytes.

\section{Electron microscopy}

Pachytene SCs

The testes of $D$. lacteum form numerous scattered islands dispersed along most of the body.

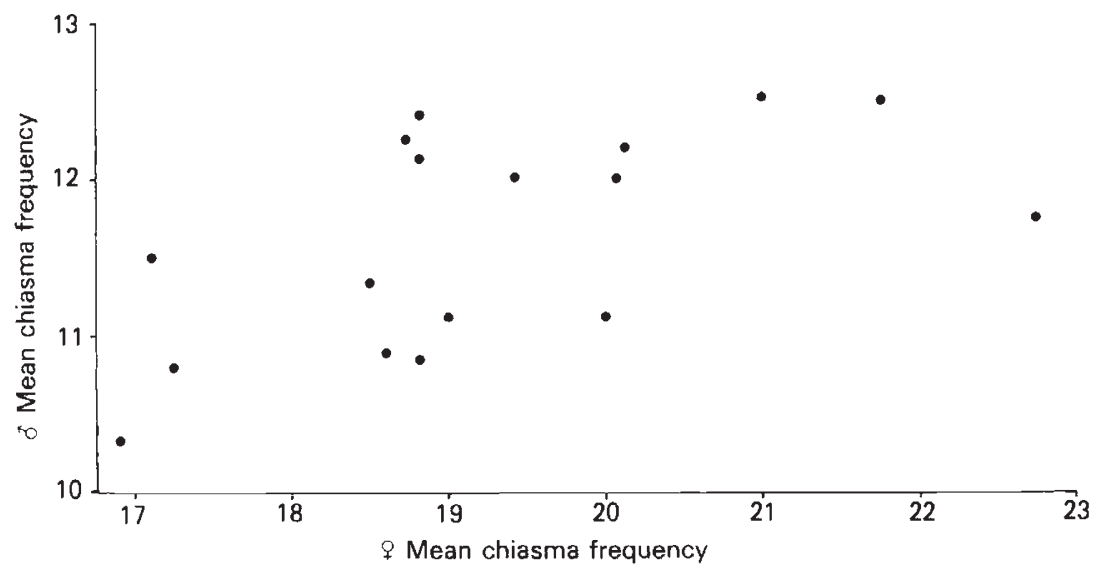

Figure 3 Spermatocyte ( () mean chiasma frequencies plotted against oocyte (\$) mean chiasma frequencies for 17 different individuals of Dendrocoelum lacteum. 
Table 2 Ranked lengths $(\mu \mathrm{m})$ of the $7 \mathrm{SCs}$ in 10 spermatocytes and 10 oocytes of Dendrocoelum lacteum at the pachytene stage, together with total SC lengths of nuclei, nuclear volumes and numbers of attached SC ends. A-D refer to four different individual animals

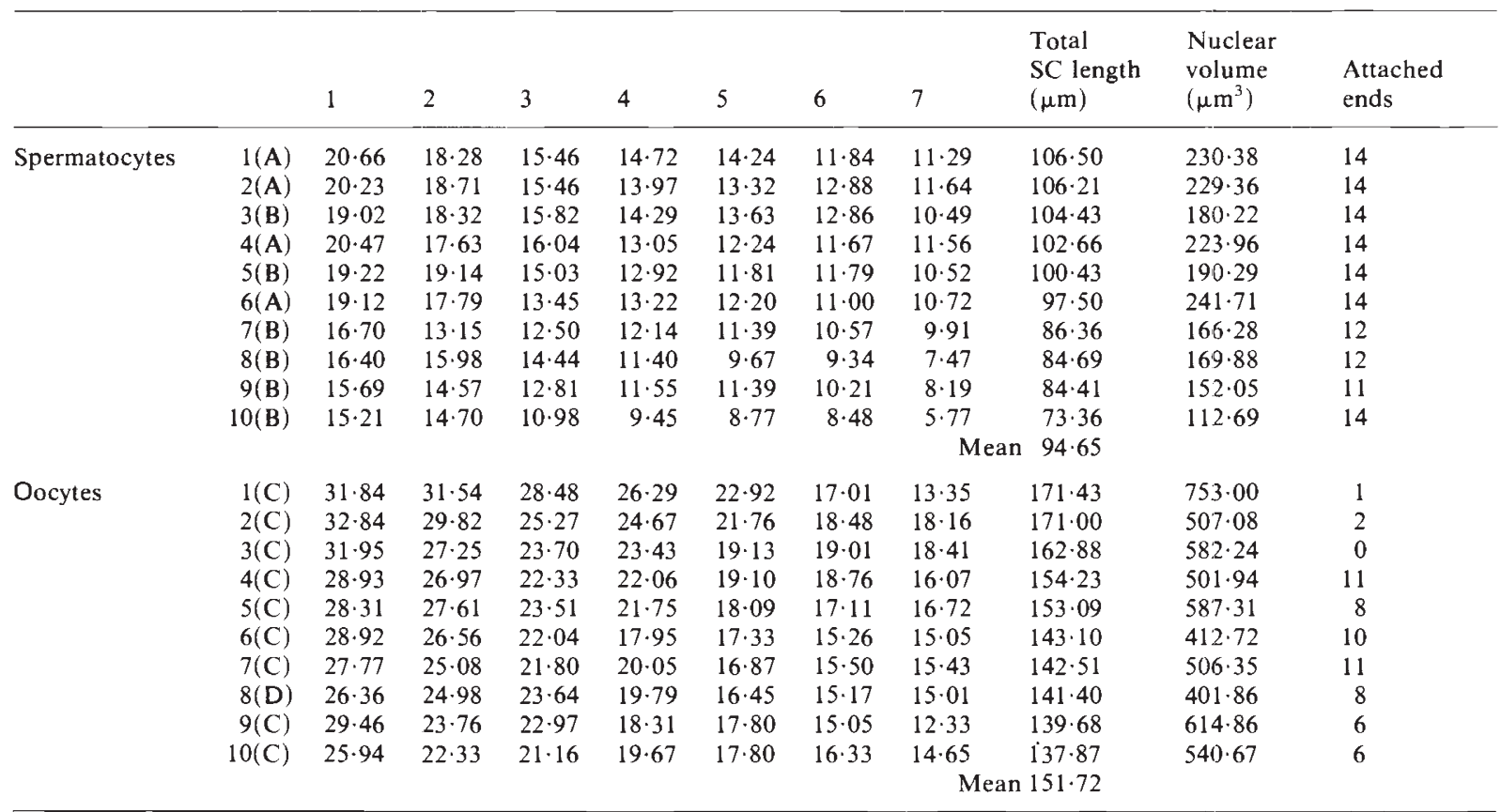

Pachytene spermatocytes occur in synchronised groups of eight cells intermingled with other spermatogenic stages in an apparently unordered manner around the central lumen of each testis island. The paired spherical ovaries lie anteriorly, directly behind the brain. Oocytes evidently show some zonation within the ovaries since there is some clustering of pachytene oocytes. However a simple linear sequence of oocyte development such as occurs in the rhabdocoel Mesostoma ehrenbergii ehrenbergii (Oakley, 1982) is not found in D. lacteum.

Spermatocyte and oocyte nuclei differ considerably in size (table 2) and general appearance (figs. 4(a) and 4(b)). Spermatocyte nuclei are relatively small $(6-8 \mu \mathrm{m}$ diameter) and have fairly evenly dispersed chromatin with some concentration around SCs, while oocyte nuclei are considerably larger $(9-11.5 \mu \mathrm{m})$ and their chromatin is heavily concentrated around SCs and separated by large chromatin-free spaces. However SC morphology is very similar in spermatocytes and oocytes and also resembles that found in another platyhelminth worm $M$. ehrenbergii ehrenbergii (Oakley and Jones, 1982; Croft and Jones, 1989). The lateral elements are indistinct and poorly differentiated from the surrounding chromatin while the pale central region contains a prominent dense central element (fig. 5).

All pachytene nuclei contain seven uninterrupted SCs, confirming that all seven chromosome pairs are fully synapsed at pachytene in both spermatocytes and oocytes. The ranges of total SC lengths in ten spermatocyte and ten oocyte pachytene nuclei are shown in table 2, together with data on nuclear volumes and numbers of attached SC ends. It is notable that spermatocytes show a significant positive linear regression of total SC length on nuclear volume $\left(F_{(1,8)}=21 \cdot 854, P<\right.$ $0.01)$ suggesting that both $\mathrm{SC}$ length and nuclear volume decrease with the progression of pachytene. However, for reasons which are not immediately apparent, oocytes do not exhibit a clear relationship between $\mathrm{SC}$ length and nuclear volume $\left(F_{(1,8)}=2 \cdot 667 ; P>0 \cdot 10\right)$. Probably one or both of these variables changes non-linearly during the progression of oocytes through pachytene. It is known, for instance, that Drosophila oocyte pachytene SCs first decrease in length, then later increase in length again (Carpenter, 1979 $a$ ). The difficulty of identifying a clear linear series of developing oocytes in Dendrocoelum ovaries hindered attempts to identify sequential changes in oocyte volume and SC length in this species. 

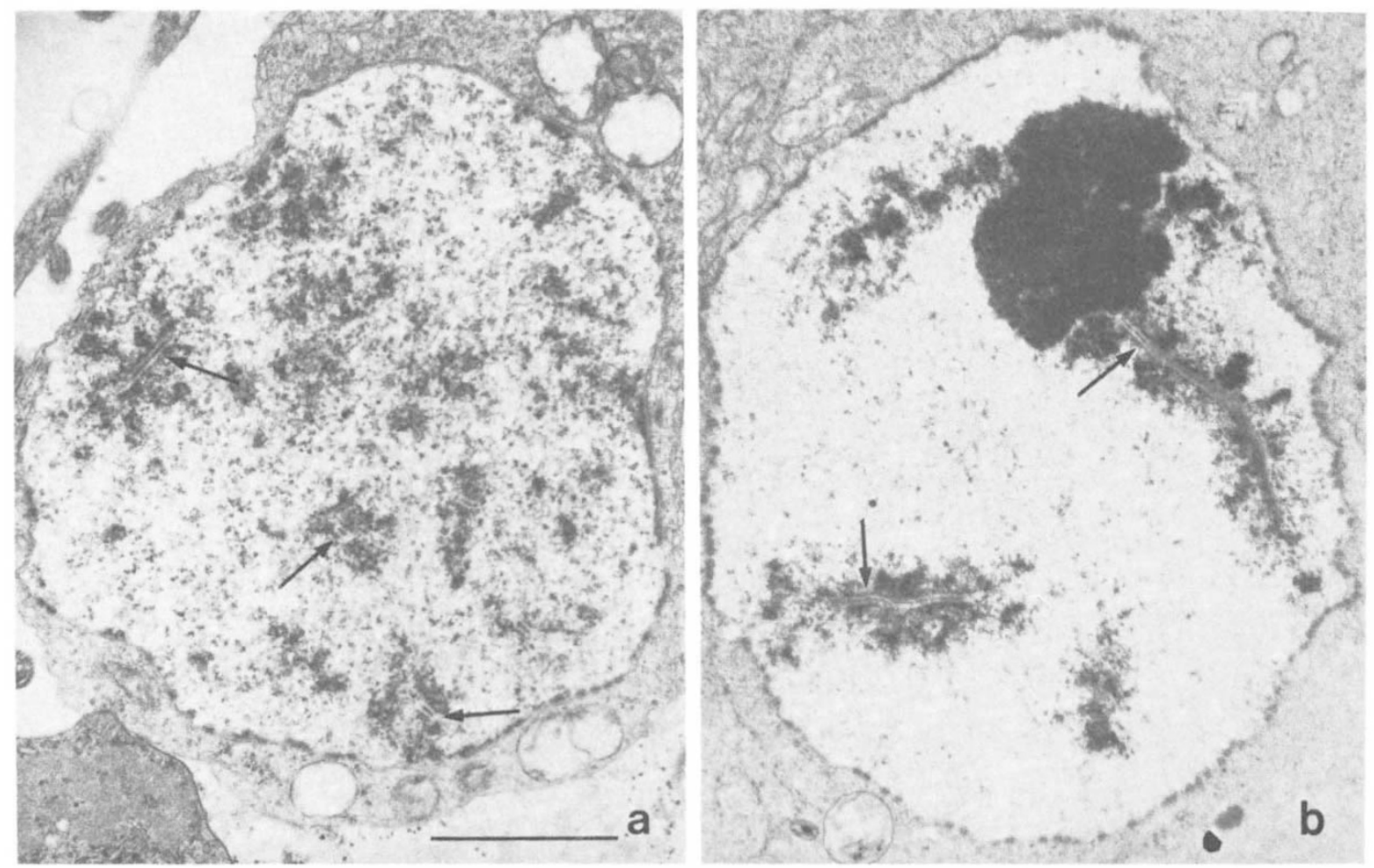

Figure 4 Low power electron micrographs of thin sections through entire spermatocyte (a) and oocyte (b) nuclei of Dendrocoelum lacteum. Arrows indicate synaptonemal complexes. $(\mathrm{Bar}=2 \mu \mathrm{m})$.

The most striking feature of the SC data is the marked difference in mean SC length between spermatocytes and oocytes. The mean SC length of spermatocyte nuclei is $94.65 \mu \mathrm{m}$ (range 73.36$106.49 \mu \mathrm{m}$ ) while in oocytes the corresponding value is $151.72 \mu \mathrm{m}$ (range $137.87-171.43 \mu \mathrm{m}$ ), that is 60 per cent longer. The ratios of longest to shortest SCs are remarkably consistent in oocytes $(1: 1 \cdot 883)$ and spermatocytes $(1: 1 \cdot 876)$ and also agree closely with the ratio of chromosome length extremes in the mitotic complement. However, the construction of an SC karytype for this species is greatly hindered by the lack of consistent and reproducible centromere structures on SCs. Although putative centromere structures are sometimes observed they do not appear with sufficient regularity and consistency even to confirm that they represent centromeres, let alone serve as an
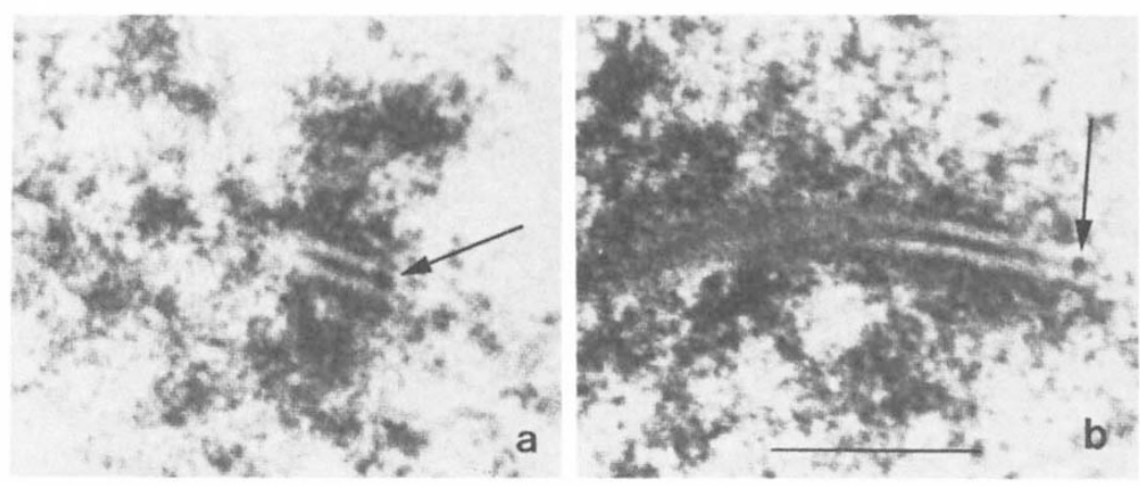

Figure 5 Details of short SC stretches containing recombination nodules (arrowed). $(\mathrm{Bar}=0 \cdot 5 \mu \mathrm{m})$. 
aid to SC identification. Individual SCs cannot be identified on the basis of length alone because of the relatively small differences in relative length between adjacent rank positions and the consequent risk of SC misclassification due to "reversals of order" (Matern and Simak, 1968; Croft and Jones, 1986).

The nucleolus organizer (NOR) serves as a marker identifying one SC in spermatocytes. The NOR is subterminally located on the second longest SC in spermatocytes, but although the mean and modal rank positions of this SC place it as the second longest in the complement, its actual rank position in individual nuclei varies between 1 and 3, which illustrates the "reversal of order" problem mentioned above. In oocytes the situation is more complex because additional nucleoli and NORs may be present, which make direct comparisons with spermatocytes difficult. Oocytes may have one or two discrete nucleoli associated with up to 4 NORs occupying subterminal locations on up to $3 \mathrm{SCs}$. Considerable variation occurs both between different animals and between oocytes from the same animal regarding the numbers of expressed NORs in oocytes.

Spermatocyte SCs show a moderate degree of bouquet polarisation. The $\mathrm{SC}$ ends are almost invariably attached to the inner surface of the nuclear envelope and the majority of ends in each nucleus are restricted in their distribution to one hemisphere of the nucleus. Only 4 per cent $\left(\frac{6}{140}\right)$ of ends were observed to be unattached in ten reconstructed pachytene spermatocytes. Bouquet polarisation is also evident in oocytes, but to a lesser extent than in spermatocytes and more SC ends are unpolarised. A surprising finding is that many SC ends $\left(\frac{56}{140}\right)$ are unattached to the nuclear envelope in oocytes, although in most of these cases the ends lie near the nuclear periphery. Individual oocyte SCs may have 0,1 or $2 \mathrm{SC}$ ends attached to the nuclear envelope. There is considerable inter-nuclear variation in the number of attached ends (table 2) which appears to bear some relation to SC length since the 3 oocyte nuclei with fewest attached ends $(0,1$ or 2$)$ also have the longest SCs.

\section{Recombination nodules}

One of the original objectives of this study was to carry out a quantitative study of recombination nodules (RNs) in spermatocytes and oocytes for comparison with chiasmata. Staining with phosphotungstic acid failed to reveal any structures which could be candidates for RNs, but after double staining with uranyl acetate and lead citrate $\mathrm{RN}$ structures were observed in both spermatocyte and oocyte pachytene nuclei. The clearest RNs seen are small dense spherical structures located directly on the central element of the SC (fig. 5). These RNs are no more than $50-70 \mathrm{~nm}$ in diameter, not much wider than the central element, and against the prominent central element characterising this species even the clearest examples are not very obvious structures. In addition to these relatively clear examples, there are many more putative RNs which are smaller, less densely stained and less distinct against the background of the dense central element. These could not be definitely identified as RNs, although some of them probably were so. This unforeseen difficulty virtually precluded any meaningful assessment of $R N$ numbers and distributions because the boundary between definite and questionable RNs was undefinable.

\section{Zygotene observations}

Unpaired axial cores (ACs) are not clearly visible against the surrounding chromatin in zygotene nuclei of $D$. lacteum, reflecting the weak differentiation of lateral elements in this species. Consequently, a full analysis of zygotene nuclei through paired and unpaired regions is not feasible. Nevertheless partial analyses can be performed, based on the numbers, lengths and distributions of identifiable SC segments present in zygotene nuclei. Table 3 gives details of four oocyte and three spermatocyte nuclei at the zygotene stage which were reconstructed and partially analysed. The total length of SC present in a zygotene nucleus, compared to the mean pachytene SC length, gives an approximate indication of the extent of pairing. Marked SC shortening between

Table 3 The pairing characteristics of 3 spermatocyte and 4 oocyte nuclei of Dendrocoelum lacteum at the zygotene stage

\begin{tabular}{|c|c|c|c|c|c|}
\hline & $\begin{array}{l}\text { SC } \\
\text { length } \\
(\mu \mathrm{m})\end{array}$ & $\begin{array}{l}\text { Per } \\
\text { cent } \\
\text { paired }\end{array}$ & $\begin{array}{l}\text { Fully } \\
\text { paired } \\
\text { SCs }\end{array}$ & $\begin{array}{l}\text { Paired } \\
\text { chromosome } \\
\text { ends }\end{array}$ & $\begin{array}{l}\text { Interstitial } \\
\mathrm{SCs}\end{array}$ \\
\hline \multicolumn{6}{|c|}{ Spermatocytes } \\
\hline 1 & $72 \cdot 45$ & $76 \cdot 6$ & 2 & 13 & 1 \\
\hline 2 & $83 \cdot 36$ & $93 \cdot 4$ & 2 & 13 & 2 \\
\hline 3 & $90 \cdot 80$ & $95 \cdot 9$ & 4 & 13 & 2 \\
\hline \multicolumn{6}{|c|}{ Oocytes } \\
\hline 1 & $73 \cdot 28$ & $48 \cdot 3$ & 1 & 5 & 3 \\
\hline 2 & $82 \cdot 34$ & $54 \cdot 3$ & 0 & 7 & 7 \\
\hline 3 & $85 \cdot 71$ & $56 \cdot 5$ & 5 & 11 & 2 \\
\hline 4 & $117 \cdot 24$ & $77 \cdot 3$ & 1 & 12 & 6 \\
\hline
\end{tabular}


zygotene and pachytene would result in considerable overestimation of pairing extent at zygotene, but this does not seem to be the case. For example, one spermatocyte zygotene nucleus with $90.8 \mu \mathrm{m}$ of SC is estimated to be 95.9 per cent paired and has four fully paired SCs and only one unpaired end, which seems reasonable. Furthermore, if SC shortening were pronounced between zygotene and pachytene then zygotene nuclei having more $\mathrm{SC}$ than the mean pachytene length are expected, but none were observed.

$\mathrm{SC}$ segments in zygotene nuclei were classified as terminal if one end was associated with the nuclear envelope, or interstitial if both ends were free and unattached. The rather large numbers of unattached chromosome ends observed in pachytene oocytes are a potential complication to this analysis. However, the detailed light microscopical analyses by Gelei (1921) clearly indicated that all chromosome ends are attached to the nuclear envelope and arranged in a bouquet during zygotene and early pachytene in oocytes; unattached ends are likely, therefore, to be a later pachytene phenomenon associated with the dissolution of the bouquet. This analysis (table 3 ) indicates that chromosome ends pair relatively early in both spermatocytes and oocytes; all nuclei with in excess of 55 per cent pairing have most (11-13) of their 14 ends paired in SCs. In addition, there is evidence of some interstitial pairing initiation in both spermatocytes and oocytes, since separate SC segments which are not attached by either end to the nuclear envelope are observed in both cell types. However, spermatocytes show relatively few interstitial SC segments, only one or two per nucleus, while oocytes show many more, between two and seven per nucleus. This may be partly explained by the fact that most of the oocyte nuclei are at a relatively early stage of zygotene, but the difference is also clearly evident in a pair of nuclei having almost identical extents of pairing. One oocyte nucleus shows $77 \cdot 3$ per cent pairing and has six interstitial SCs as well as 12 terminal SCs, whereas a spermatocyte nucleus with $76 \cdot 6$ per cent pairing has only one interstitial $\mathrm{SC}$ in addition to 13 terminal SCs.

\section{DISCUSSION}

RNs are variable in shape and size both between and within species (Carpenter, 1979b, 1984; Rasmussen and Holm, 1978). From the rather limited number of species which have been examined, it appears that the $\mathrm{RNs}$ of closely related species are more similar in size and shape than those of more distantly related species (Carpenter, 1979b). The $\mathrm{RNs}$ of $D$. lacteum are small spherical structures, not exceeding $70 \mathrm{~nm}$ in diameter. They therefore occupy the lower end of the observed range of RN sizes and are comparable to the rather small RNs which characterise many fungal species (e.g., Byers and Goetsch, 1975; Holm et al., 1981). Another platyhelminth worm, the rhabdocoel $M$. ehrenbergii ehrenbergii has, by contrast, large and very obvious RNs (Croft and Jones, 1989), which are comparable in their size and morphology to those of $D$. melanogaster oocytes. Evidently the generalisation that related species have similar sized RNs does not hold universally.

Quantifying RN populations can be problematical when RNs are uniformly small or very variable in size. This problem is not unique to $D$. lacteum and has been encountered, for example, for the small RNs of yeast (Byers and Goetsch, 1975) ellipsoid nodules of wild-type Drosophila oocytes (Carpenter, 1981), spherical and ellipsoid RNs of some meiotic mutants of Drosophila (Carpenter, 1979 b) and zygotene RNs in the fungus Coprinus (Holm et al., 1981). In D. lacteum the problem is compounded by the dense central element of the SC which makes it difficult to detect with certainty any but the largest and most prominent RNs.

Oocytes and spermatocytes of D. lacteum show a striking difference in SC length which almost exactly parallels their mean chiasma frequencies. Oocyte SCs are on average 60 per cent longer than spermatocyte $\mathrm{SCs}$ and the mean chiasma frequency of oocytes exceeds that of spermatocytes by 66 per cent. A pronounced sex difference in SC length has also been reported in humans, where oocytes have a mean SC length per nucleus which is twice that observed in spermatocytes. There is however some disagreement as to whether or not this coincides with a sex difference in chiasma and recombination frequency (Bojko, 1985; Wallace and Hulten, 1985). In the mouse, where reliable chiasma data exist for both males and females, a good correlation of SC length and chiasma frequency exists. Despite some variation due to age and interstrain differences, chiasma frequencies in male and female mice are very similar (Speed, 1977) and SC lengths measured from oocytes and spermatocytes are also alike (Speed, 1982; Moses \& Poorman, 1984). In maize, extra heterochromatic knobs on chromosomes 10 and 9 have been found to have parallel effects on recombination frequencies and SC lengths (Gillies et al., 1973; Mogensen, 1977). The study by 
Mogensen (1977) on the effects of a heterochromatic knob on the short arm of chromosome 9 is especially pertinent since higher female and lower male crossover frequencies in the short arm of chromosome 9 were accompanied by longer and shorter SCs, respectively, for that arm but not elsewhere in the genome. Finally, a difference in $\mathrm{SC}$ length which parallels a chiasma frequency difference has been observed in spermatocytes of two locust species, Locusta migratoria and Schistocerca gregaria (Croft and Jones, 1986) but this case is rather different to the previous sexdifference cases since the SC length and chiasma frequency differences are associated with a 49 per cent difference in genomic DNA amount between these species.

Correlations between SC length and recombination frequency suggest, but do not prove that they are causally related. The assertion by Mogensen (1977) that "different lengths of the SC are responsible for the sex differences in crossover frequencies" involves a further assumption about the direction of causality. This assumption may be judged to be reasonable in the light of our present knowledge of the timing of recombination in relation to SC formation. It is certainly more difficult to imagine the reverse situation, that is an effect of recombination frequency on $\mathrm{SC}$ length, but that possibility cannot be excluded. The suggestion by Mogensen (1977), based on an earlier proposal by Stern et al. (1975), that longer SCs are likely to entrap more "preselected" DNA sequences which in turn will enhance the probability of recombination occurring in that region is interesting and reasonably plausible but necessarily conjectural. This points to the need for a better understanding of the precise role of the $\mathrm{SC}$ in mediating and regulating recombination events.

Information on the organization of chromosome pairing at zygotene, in terms of the numbers and distributions of initiations and the progression of synapsis, is still relatively sparse. Among animal species, small chromosomes tend to initiate pairing nearly exclusively at the ends (e.g., Rasmussen and Holm, 1978, 1982) while larger chromosomes may, in addition, have one or more interstitial initiations (Jones and Croft, 1986). It is reasoned that additional interstitial initiations are an adaptation to facilitate the rapid and efficient pairing of larger chromosomes. Although oocytes and spermatocytes of $D$. lacteum have identical DNA amounts, the chromosome material is organized differently to give much longer SCs in oocytes. The inference from the analysis of reconstructed zygotene nuclei that early pairing occurs predominantly from chromosome ends is in broad agreement with the classical light microscopical observations (Gelei, 1921). Limited data are presented which indicate that interstitial pairing initiations may be more frequent in oocytes than in spermatocytes. Their function is presumably to facilitate efficient pairing of the relatively longer chromosomes of oocytes.

Acknowledgement We thank Dr Adelaide T. C. Carpenter for reading the manuscript and making several perceptive comments and helpful suggestions. This work was supported by a research grant $(\mathrm{GR} / \mathrm{C} / 98368)$ from the Science and Engineering Research Council.

\section{REFERENCES}

AEPPli, E. 1951. Die Chromosomenverhaltnisse bei Dendro coelum infernale (Steinmann). Ein Beitrage zur Polyploidie in Tierreich. Rev. Suisse Zool, 58, 511-518.

ARNOLD, G. 1909. The prophase in the oogenesis and the spermatogenesis of Planaria lactea O.F.M. (Dendrocoelum lacteum Oerst.). Arch. Zellforsch., 3, 431-446.

BALL, I. R. AND REYNOLDSON, T. B. 1981. British Planarians. Synopses of the British Fauna, 19. Edited by D. M. Kermack and R. S. K. Barnes. Cambridge University Press.

BENAZZI, M. AND BENAZZI LeNTATI, G. 1976. Animal Cytogenetics. Edited by B. John, H. Bauer, S. Brown, H. Kayano, A. Levan, M. White. Vol. 1: Platyhelminthes. Gebruder Borntraeger, Berlin.

BENAZZI, M. AND POCHINI, N. 1959. Alcune osservazioni citologische sulla planaria Dendrocoelum lacteum (O. F. Muller). Boll. Zool, 26, 435-444.

BOJKO, M. 1985. Human meiosis. IX. Crossing over and chiasma formation in oocytes. Carlsberg Res. Commun. $50,43-72$.

BYERS, B. AND GOETSCH, L. 1975. Electron microscopic observations on the meiotic karyotype of diploid and tetraploid Saccharomyces cerevisiae. Proc. Natl Acad. Sci. USA, 72, 5056-5060.

CARPENTER, A. T. C. $1979 a$. Synaptonemal complex and recombination nodules in wild-type Drosophila melanogaster females. Genetics, 92, 511-541.

CARPENTER, A. T. C. 1979 b. Recombination nodules and synaptonemal complex in recombination-defective females of Drosophila melanogaster. Chromosoma, 75, 259-272.

CARPENTER, A. T. C. 1981. EM autoradiographic evidence that DNA synthesis occurs at recombination nodules during meiosis in Drosophila melanogaster females. Chromosoma, $83,59-80$.

CARPENTER, A. T. C. 1984. Recombination nodules and the mechanism of crossing-over in Drosophila. Controlling Events in Meiosis. Society for Experimental Biology Symposium 28. Edited by C. W. Evans and H. G. Dickinson. Company of Biologists Ltd., Cambridge.

CROFT, J. A. AND JONES, G. H. 1986. Surface spreading of synaptonemal complexes in locusts. I. Pachytene observations. Chromosoma, 93, 483-488.

CROFT. J. A. AND JONES, G. H. 1989. Meiosis in Mesostoma ehrenbergii ehrenbergii. IV. Recombination nodules of spermatocytes; a test of the correspondence of late recombination nodules and chiasmata. Genetics, 121, 255-262.

DAHM, A. G. 1961. Cytotaxonomical analyses of four Dendrocoelum species. Lunds Universitets Arsskrift N.F. Avd. $2,57,1-44$. 
GELEI, J. 1913. Uber die Ovogenese von Dendrocoelum lacteum. Arch. Zellforsch., 11, 51-150.

GeleI, J. 1921. Die Langskonjugation der Chromosomen. Arch. Zellforsch., 16, 52-130.

GELEI, J. 1922. Die Konjugationsfrage der Chromosomen in der Literatur und meine Befunde. Arch. Zellforsch., 16, $300-370$

GILLIES, C. B., RASMUSSEN, S. W. AND WETTSTEIN D. VON. 1973. The synaptinemal complex in homologous and nonhomologous pairing of chromosomes. Cold Spring Harbor Symp. Quant. Biol., 38, 117-122.

HOLM, P. B., RASMUSSEN, S. W., ZICKLER, D., LU, B. C. AND SAGE, J. 1981. Chromosome pairing, recombination nodules and chiasma formation in the Basidiomycete Coprinus cinereus. Carlsberg Res. Commun., 46, 305-346.

JONES, G. H. AND CROFT, J. A. 1986. Surface spreading of synaptonemal complexes in locusts. II. Zygotene pairing behaviour. Chromosoma, 93, 489-495.

MATERN, B. AND SIMAK, M. 1968. Statistical problems in karyotype analysis. Hereditas, 59, 280-288.

MATTHIESON, E. 1904. Ein Beitrag zur Embryologie der Susswasserdendrocoelen. Z. wiss. Zool., 77, 274-361.

MELANDER, Y, 1963. Cytogenetic aspects of embryogenesis in Paludiocola, Tricladida. Hereditas, 49, 119-166.

MOGENSEN, H. L. 1977. Ultrastructural analysis of female pachynema and the relationship between synaptonemal complex length and crossing-over in Zea mays. Carlsberg Res. Commun., 42, 475-497.

MOSES, M. J. AND POORMAN, P. A. 1984. Synapsis, synaptic adjustment and DNA synthesis in mouse oocytes. Chromosomes Today 8. Proceedings of the 8th International Chromo some Conference. Edited by M. D. Bennett, A. Gropp and U. Wolf, George Allen and Unwin, London, pp. 90-103.
OAKLEY, H. A. 1982. Meiosis in Mesostoma ehrenbergii ehrenbergii (Turbellaria, Rhabdocoela) II. Synaptonemal complexes, chromosome pairing and disjunction in achiasmate oogenesis. Chromosoma, 87, 133-147.

OAKLEY, H. A. AND JONES, G. H. 1982. Meiosis in Mesostoma ehrenbergii ehrenbergii (Tubellaria, Rhabdocoela) 1. Chromosome pairing, synaptonemal complexes and chiasma localisation in spermatogenesis. Chromosoma, 85 , 311-322.

PASTOR, J. B. AND CALLAN, H. G. 1952. Chiasma formation in spermatocytes and oocytes of the Turbellarian Dendro coelum lacteum. J. Genet., 50, 449-454.

RASMUSSEN, S. W. AND HOLM, P. B. 1978. Human meiosis. 11. Chromosome pairing and recombination nodules in human spermatocytes. Carlsberg Res. Commun., 43, 275-327.

RASMUSSEN, S. W. AND HOLM, P. B. 1982. The meiotic prophase in Bombyx mori. Insect ultrastructure. Edited by R. C. King and H. Akai. Plenum, New York, London, pp. 61-85.

SPEED, R. M. 1977. The effects of ageing on the meiotic chromosomes of male and female mice. Chromosoma, 64, 241-254.

SPEED, R. M. 1982. Meiosis in the foetal mouse ovary. I. An analysis at the light microscope level using surface spreading. Chromosoma, 85, 427-437.

STERN, H., WESTERGAARD, M. AND WETTSTEIN, D. VON. 1975 Presynaptic events in meiocytes of Lilium longiflorum and their relation to crossing-over. A preselection hypothesis Proc. Natl Acad. Sci. USA, 72, 961-965.

WALI.ACE, B. M. N. AND HULTEN, M. 1985. Meiotic chromosome pairing in the normal human female. Ann. Hum. Genet., 49, 215-226.

WELLS, B. 1974. A convenient technique for the collection of ultra-thin serial sections. Micron, 5, 79-81. 